\section{Uso e necessidade de prótese dentária em idosos brasileiros segundo a Pesquisa Nacional de Saúde Bucal (SBBrasil 2010): prevalências e fatores associados}

\author{
Needs for dental prostheses and their use in \\ elderly Brazilians according to the National Oral \\ Health Survey (SBBrazil 2010): prevalence rates \\ and associated factors
}

\section{Uso y necesidad de prótesis dentales en ancianos brasileños, según la Encuesta Nacional de Salud Bucal (SBBrasil 2010): prevalencias y factores asociados}

Juliana S. Azevedo 1

Marina Sousa Azevedo 1

Luisa Jardim Correa de Oliveira 1

Marcos Britto Correa 1

Flávio Fernando Demarco 1

doi: 10.1590/0102-311X00054016

\title{
Resumo
}

O objetivo deste estudo foi avaliar o uso e a necessidade de prótese dentária entre os idosos brasileiros (65-74 anos) e verificar fatores associados. Foram analisados dados de 7.496 idosos participantes do Pesquisa Nacional de Saúde Bucal realizado em 2010 (SBBrasil 2010). O uso e a necessidade de prótese dentária foram usados como desfechos. As variáveis de exposição incluíram características demográficas, socioeconômicas, de uso de serviços e de autopercepção da saúde bucal. Análises descritiva, bivariada e multivariada foram realizadas. A prevalência de uso e necessidade de prótese dentária foi de 78,2\% e 68,7\%, respectivamente. A Região Nordeste foi a que apresentou a menor prevalência de uso $(71,3 \%)$ e a maior de necessidade prótese dentária (82,9\%). As análises multivariadas por meio de regressão de Poisson revelaram maior uso de prótese dentária em mulheres, naqueles com 5 a 7 anos de estudos e nos que foram a serviço particular, e houve menor uso em indivíduos pretos e com necessidade autorreferida de prótese dentária. Menor necessidade de prótese dentária foi observada em mulheres e nos usuários de serviço particular, e maior naqueles que autorreferiram necessidade de prótese dentária. Diferenças regionais foram observadas na distribuição do uso e da necessidade de prótese dentária. Ainda assim, os achados revelaram altas prevalências de ambos os desfechos em todas as regiões. Variáveis socioeconômicas, demográficas e de uso de serviços influenciaram a ocorrência de uso e de necessidade de prótese dentária.

Prótese Dentária; Inquéritos de Saúde; Saúde Bucal; Idoso

\author{
Correspondência \\ F. F. Demarco \\ Programa de Pós-graduação em Epidemiologia, Universidade \\ Federal de Pelotas. \\ Rua Marechal Deodoro 1160, 3o piso, sala 321, Pelotas, RS \\ 96020-220, Brasil. \\ ffdemarco@gmail.com \\ 1 Universidade Federal de Pelotas, Pelotas, Brasil.
}




\section{Introdução}

Globalmente tem sido observada uma transição demográfica, caracterizada pelo envelhecimento populacional, decorrente da redução da taxa de fecundidade, menor mortalidade e do aumento da expectativa de vida, estimando-se que entre 2010 e 2050 a proporção de idosos duplicará, acontecendo este processo de maneira mais intensa nos países em desenvolvimento ${ }^{1}$. No Brasil, o envelhecimento populacional tem sido particularmente rápido, com os idosos aumentando de $11 \%$ da população economicamente ativa em 2005 para 49\% em 2050 2. A rápida velocidade com que isso vem ocorrendo no país determina a necessidade de mudanças para lidar com esse novo perfil epidemiológico. Nessas mudanças está incluída a saúde bucal, com a necessidade de modificações no âmbito dos serviços do Sistema Único de Saúde (SUS) em relação às necessidades de tratamento dessa população 3.

Dentre as principais alterações bucais encontradas nos idosos estão as perdas dentárias e o edentulismo (perda total dos dentes) 4 . A perda dental severa afeta 2,3\% da população mundial 5 , sendo também marcadora da desigualdade social 6,7. As perdas dentárias causam prejuízo na mastigação, na digestão, na fonação e na estética, o que favorece o desenvolvimento de distúrbios psicológicos e causa impacto negativo na qualidade de vida 6,8,9. Além disso, estudos apontam relação entre perda dentária e obesidade, déficit nutricional, hipertensão, disfunção cognitiva e maior risco de mortalidade 10,11,12,13. Portanto, devido às altas prevalências, acrescido ao dano causado para os indivíduos e o elevado custo para o tratamento, as perdas dentárias são consideradas um problema de saúde pública, com maior ocorrência nas parcelas mais desfavorecidas socialmente da população 12 . O uso de prótese dentária é indicado para a recuperação da capacidade mastigatória, para a melhora do aspecto estético e de fonação dos indivíduos acometidos pela perda dental, impactando na qualidade de vida dos mesmos 14 .

Uma das metas globais da Organização Mundial da Saúde (OMS) para o ano 2000 era de que houvesse pelo menos 50\% dos indivíduos de 65-74 anos com 20 ou mais dentes na boca 15 . O uso e a necessidade de prótese são investigados baseando-se em índice desenvolvido pela própria OMS, que determinada a necessidade considerando as perdas dentárias existentes nos arcos superior e inferior, sendo o uso computado pela presença de um ou mais tipos de prótese em um ou nos dois arcos. O levantamento nacional de 2003 (Condições de Saúde Bucal da População Brasileira - SBBrasil 2003) revelou que apenas 10,3\% dos brasileiros dessa faixa etária possuíam 20 ou mais dentes presentes 6 , e em 2010 esse percentual mudou para 11,5\% 16. Apesar de ocorrer uma redução importante das perdas dentárias entre os indivíduos mais jovens, entre os mais velhos isto não vem acontecendo 16, indicando um acúmulo da necessidade de prótese dentária nestas populações. Portanto, os resultados do último levantamento tornaram evidentes as necessidades de ampliar a oferta de ações de maior complexidade, como reabilitação protética, a fim de suprir a demanda reprimida no país. Essa oferta vem aumentando desde 2004 com o lançamento da Política Nacional de Saúde Bucal, por intermédio dos Centros de Especialidades Odontológicas (CEO) e dos Laboratórios Regionais de Prótese Dentária (LRPD) 17. Entretanto, ainda não está elucidado o impacto que essa política pode ter causado no status protético da população idosa brasileira. Da mesma forma, conhecer os resultados decorrentes dessas mudanças que aconteceram no Brasil pode ser importante para a aplicação de medidas similares em outros países.

Desse modo, o objetivo deste trabalho foi avaliar o uso e a necessidade de prótese dentária na população idosa brasileira, e verificar as suas associações com fatores socioeconômicos e demográficos, necessidade de tratamento autorreferida e local de uso do serviço odontológico, além de averiguar as diferenças entre as macrorregiões do Brasil.

\section{Metodologia}

Os dados utilizados neste estudo foram disponibilizados pela Divisão Nacional de Saúde Bucal do Ministério da Saúde, após preenchimento e envio de um formulário e de um termo de compromisso dos pesquisadores envolvidos na proposta. O SBBrasil 2010 (Pesquisa Nacional de Saúde Bucal, realizado em 2010) foi submetido ao Comitê de Ética em Pesquisa do Ministério da Saúde, foi aprovado e recebeu registro na Comissão Nacional de Ética em Pesquisa (CONEP) do Conselho Nacional de Saúde, sob o no 15.498. Todos os participantes assinaram um Termo de Consentimento Livre e Esclarecido. 
Trata-se de um estudo transversal, que utilizou dados secundários do banco de dados da SBBrasil realizada no ano de 2010 relativos aos idosos de 65-74 anos. Essa pesquisa é um inquérito epidemiológico de saúde bucal de abrangência nacional, com representatividade para as capitais dos estados e Distrito Federal, para as cinco macrorregiões naturais e também para as cidades do interior de cada macrorregião 18, de acordo com a divisão do Instituto Brasileiro de Geografia e Estatística (IBGE): Norte, Nordeste, Centro-oeste, Sul e Sudeste.

A amostra foi selecionada por meio de método probabilístico de conglomerados. Esse processo de amostragem foi estruturado em dois estágios para as capitais: setor censitário e município, e em três estágios para o interior das cinco macrorregiões: município, setor censitário e domicílio. Para o grupo etário dos idosos, adotou-se o coeficiente de variação como indicador de precisão para estimativas de prevalências. Escolheu-se o número mínimo de entrevistas esperando-se que as prevalências estimadas fossem maiores que $10 \%$ e que seus erros padrão não ultrapassassem $15 \%$ destes valores. Assim, em cada capital e no interior das cinco macrorregiões estimou-se entrevistar 250 indivíduos, variando o número de residências segundo o tamanho de cada cidade ou região. Maior detalhamento pode ser encontrado em outra publicação sobre o desenho metodológico da pesquisa 18 . A amostra foi composta por 7.509 idosos residentes nas 26 capitais, no Distrito Federal e em mais de 30 municípios do interior de cada região, totalizando 177 municípios.

A metodologia usada com relação aos dados de saúde bucal seguiu as recomendações da OMS 19, com algumas características que foram adequadas à realidade do país e aprimoradas do projeto anterior ${ }^{18}$. Foram feitas visitas domiciliares para a realização dos exames bucais e entrevistas, sendo que mais detalhes podem ser encontrados em outros estudos 16,18 .

Os desfechos avaliados no presente trabalho foram o uso e a necessidade de prótese dentária da população idosa seguindo os critérios da OMS, os quais levam em consideração a presença de espaços protéticos e sua reabilitação 19 . Os dois índices não são excludentes, ou seja, um mesmo indivíduo poderia estar usando e, ao mesmo tempo, necessitando de prótese(s).

Para o uso de prótese dentária os seguintes critérios foram considerados: (0) não usa prótese dentária; (1) usa uma ponte fixa; (2) usa mais de uma ponte fixa; (3) usa uma prótese parcial removível; (4) usa ponte fixa e prótese parcial removível combinadas; (5) usa prótese total. Para necessidade de prótese dentária os critérios foram: (0) não necessita; (1) necessita uma prótese dentária para substituir um dente; (2) necessita uma prótese dentária para substituir mais de um dente; (3) necessita uma combinação de prótese dentária para substituir mais de um dente; (4) necessita uma prótese total.

Os critérios avaliados nas próteses em uso para estabelecer se estavam inadequadas e necessitavam ser trocadas foram: retenção (se estava folgada ou apertada); estabilidade e reciprocidade (se apresentava deslocamento ou báscula); fixação (se causava lesão aos tecidos); e estética (se apresentava manchas ou fraturas e não estava adequada ao perfil facial do paciente). Quando pelo menos uma dessas condições estava presente, considerava-se haver necessidade de prótese. Para análise das associações, tanto o uso como a necessidade de prótese, que avaliam o tipo de prótese que o indivíduo usa ou necessita, foram dicotomizados pelos autores ("0" não; "1" sim).

As variáveis socioeconômicas e demográficas obtidas no SBBrasil 2010 foram categorizadas pelos autores da seguinte forma: renda familiar (menos de $\mathrm{R} \$ 500,00$, de $\mathrm{R} \$ 501,00$ a $\mathrm{R} \$ 1.500,00$, de $\mathrm{R} \$$ 1.501 a $R \$ 2.500$, maior que $R \$ 2.500,00$ ), grau de escolaridade categorizado conforme anos de estudos (até 4 anos de estudos, de 5-7 anos, 8 ou mais anos), local onde foi a última consulta odontológica (público, particular/convênio, outros, nunca consultou com dentista, não informado). Foram ainda utilizadas na categorização original do levantamento as variáveis sexo (feminino e masculino), cor/ raça (brancos, pardos, pretos, amarelos e indígenas) e autopercepção em relação à necessidade de usar ou trocar a prótese que o indivíduo estava usando. Essa última por meio da pergunta: "O sr(a) considera que necessita usar prótese total (dentadura) ou trocar a que está usando atualmente?”, que foi categorizada em não, sim e não sei.

Os dados do levantamento foram organizados pela coordenação nacional do estudo em um banco de dados, utilizando-se o software EpiData (Epidata Assoc., Odense, Dinamarca). O banco de dados fornecido foi transferido para o software Stata 12.0 (StataCorp LP, College Station, Estados Unidos), no qual os autores realizaram análise estatística do presente trabalho. Todas as análises usaram o prefixo svy considerando-se o plano complexo de amostragem e pesos amostrais. 
Análise descritiva foi realizada para avaliar a distribuição das variáveis de uso e necessidade de prótese por macrorregião. Calculou-se a prevalência dos desfechos, conforme as variáveis independentes, e a seguir, realizou-se análise bivariada por meio do teste do qui-quadrado de Rao-Scott. Análise multivariada usando-se a regressão de Poisson foi realizada a fim de estimar as razões de prevalência bruta e ajustada e seus intervalos de 95\% de confiança (IC95\%). O método de seleção das variáveis no modelo final foi o stepwise com seleção para trás. Foram mantidas no modelo final apenas as variáveis que apresentaram valor de $\mathrm{p}<0,25$.

\section{Resultados}

A amostra foi constituída por todos os indivíduos com dados coletados para as variáveis de interesse, totalizando 7.496 idosos. A prevalência do uso de prótese foi de 78,2\% e de necessidade de prótese foi de $68,7 \%$. Considerando-se as macrorregiões, as prevalências de uso e necessidade de prótese dentária foram, respectivamente: Norte 76,2\% e 80,6\%; Nordeste 71,3\% e 82,9\%; Sudeste 77,8\% e 66,8\%; Sul 86\% e 60,4\%; e Centro-oeste 73,5\% e 77\%. Na Tabela 1, estão os resultados sobre o uso e a necessidade de prótese dentária por arcos dentários nas cinco macrorregiões do Brasil. Com relação ao uso de prótese nos arcos superior e inferior, em todas as regiões o uso é maior no arco superior e as prótese dentária mais utilizadas são as prótese totais e próteses parcial removíveis. Diferenças regionais são evidentes em relação ao uso de prótese dentária. A maior prevalência de uso foi encontrada na macrorregião Sul (86\%) e a menor na macrorregião Nordeste (71,3\%). A necessidade de prótese dentária também foi mais prevalente no arco superior do que no inferior e o tipo de prótese dentária de maior necessidade é a prótese total. Também foram encontradas diferenças regionais em relação à prevalência da necessidade de prótese dentária, sendo as maiores na macrorregião Nordeste $(82,9 \%)$ seguida da macrorregião Norte (80,6\%). Analisando a ocorrência de uso e de necessidade de prótese, que pode ser concomitante, observa-se que a maioria dos idosos tanto usa como necessita de prótese dentária (48,3\%). O uso de prótese sem necessidade foi observado em 29,9\% dos idosos, e 20,4\% apresentavam necessidade sem usar nenhum tipo de prótese dentária.

Os resultados da análise bivariada entre a prevalência do uso e necessidade de prótese dentária e as variáveis independentes estão apresentados na Tabela 2. O uso de prótese foi maior nos indivíduos do sexo feminino, cor da pele branca e usuários do serviço particular/convênio. Em relação à necessidade de tratamento autorreferido, aqueles indivíduos que acreditavam não necessitar de prótese foram os que apresentaram menor uso de prótese dentária. Já em relação à necessidade de prótese dentária houve associação com todas as variáveis independentes analisadas. A necessidade de prótese foi maior nos idosos do sexo masculino, de cor da pele preta, com renda igual ou inferior a $\mathrm{R} \$ 500,00$, que tinham até 4 anos de estudos, que referiram necessidade de tratamento quanto ao uso ou troca da prótese total (dentadura) e usuários do serviço odontológico público.

A Tabela 3 mostra os resultados da análise multivariada para os fatores associados ao uso de prótese dentária. Após ajuste para variáveis confundidoras, o sexo feminino foi associado com maior uso de prótese (RP = 1,19; IC95\%: 1,10-1,29), assim como aqueles que tinham de 5-7 anos de estudos $(\mathrm{RP}=1,11$; IC95\%: 1,03-1,19), em relação aos com menos anos de estudos. Indivíduos pretos $(\mathrm{RP}=$ 0,83; IC95\%: 0,74-0,93) e pardos ( $\mathrm{RP}=$ 0,93; IC95\%: 0,87-0,99) apresentaram menor uso de prótese quando comparados aos brancos. Ainda, indivíduos que acreditavam não necessitar de prótese apresentaram menor uso ( $\mathrm{RP}=0,81$; IC95\%: 0,76-0,87) em relação aos que acreditavam necessitar. Idosos que faziam uso de serviço particular ou de outros tipos de serviço apresentaram maior uso de prótese. Idosos que reportaram nunca ter ido ao dentista faziam mais uso de prótese do que aqueles que relataram usar o serviço público.

A Tabela 4 mostra as análises bruta e ajustada em relação aos fatores associados à necessidade de prótese. Após ajuste, a maior renda familiar ( $\mathrm{RP}=$ 0,73; IC95\%: 0,57-0,93) e o uso do serviço particular $(\mathrm{RP}=$ 0,87; IC95\%: 0,77-0,98) permaneceram associados à menor necessidade de prótese, assim como a necessidade de tratamento autorreferido que se manteve associado à maior prevalência de necessidade de prótese dentária ( $\mathrm{RP}=1,58$; IC95\%: 1,39-1,79). 
Tabela 1

Análise descritiva do uso e necessidade de prótese em idosos por arco dentário segundo macrorregião, Brasil. SBBrasil 2010 (N = 7.496).

\begin{tabular}{|c|c|c|c|c|c|c|}
\hline Uso e necessidade de prótese & $\begin{array}{c}\text { Norte } \\
\%(\text { IC95\%) }\end{array}$ & $\begin{array}{l}\text { Nordeste } \\
\%(\text { IC95\%) }\end{array}$ & $\begin{array}{l}\text { Sudeste } \\
\%(I C 95 \%)\end{array}$ & $\begin{array}{c}\text { Sul } \\
\%(\text { IC95\%) }\end{array}$ & $\begin{array}{l}\text { Centro-oeste } \\
\%(I C 95 \%)\end{array}$ & $\begin{array}{c}\text { Total } \\
\% \text { (IC95\%) }\end{array}$ \\
\hline \multicolumn{7}{|l|}{ Uso (arco superior) } \\
\hline Não usa & $26,3(21,0-32,4)$ & $29,3(25,5-33,4)$ & $22,6(17,9-28,2)$ & $14,9(10,8-20,0)$ & $26,7(21,8-32,2)$ & $22,4(19,2-26,0)$ \\
\hline 1 ponte fixa & $0,7(0,3-1,4)$ & $2,4(1,4-4,0)$ & $4,0(2,4-6,6)$ & $5,1(2,6-9,6)$ & $2,5(1,5-4,4)$ & $3,7(2,6-5,4)$ \\
\hline Mais de uma ponte fixa & $0,3(0,1-0,9)$ & $0,8(0,4-1,6)$ & $0,8(0,4-1,8)$ & $0,8(0,4-1,7)$ & $0,8(0,4-1,7)$ & $0,8(0,5-1,3)$ \\
\hline Prótese parcial removível & $6,7(4,4-10,1)$ & $8,6(7,0-10,5$ & $6,3(4,5-8,7)$ & $11,1(8,47-14,4)$ & $7,1(5,2-9,5)$ & $7,3(6,0-9,0)$ \\
\hline $\begin{array}{l}\text { Prótese parcial removível + } \\
\text { ponte fixa }\end{array}$ & $0,8(0,4-2,0)$ & $0,9(0,4-1,9$ & $1,2(0,5-3,1)$ & $1,2(0,5-2,6)$ & $1,4(0,7-2,6)$ & $1,2(0,6-2,2)$ \\
\hline Prótese total & $63,5(58,2-68,6)$ & $56,4(52,6-60,1)$ & $62,8(56,6-68,6)$ & $66,3(59,2-72,8)$ & $60,2(53,9-66,1)$ & $62,6(58,6-66,5)$ \\
\hline Sem informação & $1,6(0,8-3,5)$ & $1,6(0,7-3,4)$ & $2,2(1,0-4,8)$ & $0,7(0,2-2,4)$ & $1,3(0,7-2,4)$ & $1,8(1,0-3,4)$ \\
\hline \multicolumn{7}{|l|}{ Uso (arco inferior) } \\
\hline Não usa & $53,3(46,5-60,0)$ & $53,2(48,0-58,3)$ & $43,2(36,4-50,0)$ & $40,6(34,4-47,2)$ & $47,8(43,0-52,7)$ & $44,5(40,0-49,1)$ \\
\hline 1 ponte fixa & $0,3(0,1-0,8)$ & $1,1(0,7-1,9)$ & $1,4(0,5-3,6)$ & $2,3(1,2-4,5)$ & $1,0(0,4-2,6)$ & $1,4(0,8-2,6)$ \\
\hline Mais de uma ponte fixa & $0,1(0,0-0,2)$ & $0,7(0,4-1,2)$ & $1,0(0,4-2,6)$ & $1,1(0,4-2,8)$ & $0,5(0,2-1,1)$ & $0,9(0,5-1,8)$ \\
\hline Prótese parcial removível & $10,6(7,3-15,2)$ & $10,6(8,5-13,2)$ & $13,5(10,6-17,1)$ & $12,5(9,3-17,6)$ & $10,4(8,4-12,8)$ & $12,7(10,7-15,0)$ \\
\hline $\begin{array}{l}\text { Prótese parcial removível + } \\
\text { ponte fixa }\end{array}$ & $0,5(0,2-1,1)$ & $1,7(0,8-3,6)$ & $1,2(0,4-3,3)$ & $0,7(0,3-1,9)$ & $1,5(0,7-3,2)$ & $1,2(0,6-2,3)$ \\
\hline Prótese total & $33,9(29,6-38,5)$ & $31,2(26,9-35,2)$ & $37,6(31,8-43,8)$ & $41,9(35,9-48,2)$ & $37,47(33,0-42,1)$ & $37,5(33,6-41,5)$ \\
\hline Sem informação & $1,3(0,6-2,9)$ & $1,6(0,7-3,4)$ & $2,1(1,0-4,5)$ & $0,7(0,2-2,4)$ & $1,4(0,8-2,4)$ & $1,8(1,0-3,2)$ \\
\hline \multicolumn{7}{|l|}{ Necessidade (arco superior) } \\
\hline Não necessita & $32,9(28,0-38,2)$ & $27,9(23,2-33,2)$ & $44,8(36,7-53,2)$ & $52,1(44,6-63,3)$ & $32,1(27,1-37,6)$ & $43,3(37,8-48,8)$ \\
\hline Substituição de 1 elemento & $0,2(0,1-0,5)$ & $2,2(1,3-3,7)$ & $2,3(1,0-5,2)$ & $2,3(1,0-5,2)$ & $1,0(0,5-2,1)$ & $2,1(1,5-3,1)$ \\
\hline Mais de 1 elemento & $9,9(7,4-13,2)$ & $13,9(11,7-16,5)$ & $8,3(5,6-12,1)$ & $7,9(5,4-11,5)$ & $10,9(8,0-14,6)$ & $9,0(7,1-11,4)$ \\
\hline Combinação de próteses & $3,8(2,1-6,7)$ & $6,7(4,9-9,1)$ & $3,2(1,8-5,6)$ & $2,3(1,2-4,2)$ & $5,7(3,6-8,9)$ & $3,6(2,5-5,0)$ \\
\hline Prótese total & $51,9(44,7-58,9)$ & $47,5(41,0-54,1)$ & $39,3(31,0-48,3)$ & $32,7(24,8-41,7)$ & $49,3(43,3-55,5)$ & $40,3(34,7-46,1)$ \\
\hline Sem informação & $1,3(0,6-2,9)$ & $1,7(0,8-3,5)$ & $2,1(1,0-4,5)$ & $0,7(0,2-2,4)$ & $1,0(0,5-2,0)$ & $1,7(1,0-3,2)$ \\
\hline \multicolumn{7}{|l|}{ Necessidade (arco inferior) } \\
\hline Não necessita & $20,8(17,2-24,9)$ & $19,2(15,6-23,3)$ & $35,0(27,6-43,3)$ & $41,5(34,0-49,3)$ & $25,1(20,8-30,0)$ & $33,2(28,2-38,6$ \\
\hline Substituição de 1 elemento & $1,2(0,5-2,8)$ & $2,2(1,5-3,3)$ & $1,7(0,6-4,4)$ & $3,6(1,5-7,9)$ & $2,2(1,3-3,7)$ & $2,0(1,1-3,5)$ \\
\hline Mais de 1 elemento & $25,3(20,6-30,7)$ & $24,4(21,4-27,7)$ & $20,2(15,5-25,8)$ & $23,7(18,5-29,9)$ & $22,1(16,39-28,5)$ & $21,6(18,4-25,1)$ \\
\hline Combinação de próteses & $8,1(5,4-12,0)$ & $10,8(8,1-14,2)$ & $7,4(5,0-10,8)$ & $6,1(3,6-10,2)$ & $7,4(4,5-11,9)$ & $7,5(5,8-9,7)$ \\
\hline Prótese total & $43,3(37,1-49,7)$ & $41,7(35,2-48,5)$ & $33,6(27,5-41,3)$ & $24,5(18,2-32,1)$ & $42,1(35,8-48,7)$ & $33,9(29,3-38,9)$ \\
\hline Sem informação & $1,3(0,6-2,9)$ & $1,7(0,8-3,5)$ & $2,2(1,0-4,6)$ & $0,7(0,2-2,4)$ & $1,0(0,5-2,0)$ & $1,8(1,0-3,2)$ \\
\hline
\end{tabular}

IC95\%: intervalo de 95\% de confiança.

\section{Discussão}

Este trabalho revelou que aproximadamente $3 / 4$ da população idosa brasileira apresentam uso e necessidade de prótese dentária em consequência do alto índice de perdas dentárias, cuja prevalência permanece alta desde o levantamento nacional anterior 6 . Além disso, a ocorrência de uso e necessidade de prótese dentária apresentaram diferenças regionais, estando associadas a características socioeconômicas, demográficas e de uso de serviço. O país chega a apresentar $54 \%$ de edentados totais, diferindo dos idosos de países desenvolvidos como França e Noruega que apresentam apenas 16\% 16. Percebe-se que a prevalência e a distribuição das perdas dentárias variam significantemente segundo os níveis de desenvolvimento dos países, sendo esta variação associada a uma combinação de fatores individuais, culturais e socioeconômicos 3,20. 


\section{Tabela 2}

Prevalência do uso e necessidade de prótese em idosos de 65-74 anos de idade e fatores associados. SBBrasil 2010 (N = 7.496 ).

\begin{tabular}{|c|c|c|c|c|c|}
\hline Variável & $\begin{array}{c}\text { Total da } \\
\text { amostra (\%) }\end{array}$ & $\begin{array}{c}\text { Prevalência de } \\
\text { uso de prótese (\%) }\end{array}$ & Valor de $\mathrm{p}$ & $\begin{array}{c}\text { Prevalência de } \\
\text { necessidade de } \\
\text { prótese }(\%)\end{array}$ & Valor de $p$ \\
\hline \multicolumn{6}{|c|}{ Características socioeconômicas e demográficas } \\
\hline Sexo & & & $<0,001$ & & 0,008 \\
\hline Masculino & 37,8 & 68,6 & & 73,4 & \\
\hline Feminino & 62,2 & 84,0 & & 65,8 & \\
\hline Raça/Cor & & & $<0,001$ & & 0,008 \\
\hline Branca & 54,7 & 83,4 & & 65,3 & \\
\hline Preta & 13,9 & 64,4 & & 77,0 & \\
\hline Amarela & 1,3 & 72,8 & & 73,6 & \\
\hline Parda & 29,5 & 75,4 & & 71,5 & \\
\hline Indígena & 0,6 & 76,5 & & 37,2 & \\
\hline Renda familiar (em Reais) & & & 0,092 & & $<0,001$ \\
\hline$\leq 500$ & 13,5 & 69,2 & & 78,4 & \\
\hline $501-1.500$ & 56,6 & 79,0 & & 70,9 & \\
\hline $1.501-2.500$ & 18,2 & 82,5 & & 63,7 & \\
\hline$>2.500$ & 11,7 & 79,3 & & 51,5 & \\
\hline Anos de estudos & & & 0,055 & & 0,038 \\
\hline Até 4 & 62,5 & 76,4 & & 71,5 & \\
\hline $5-7$ & 15,3 & 85,8 & & 69,2 & \\
\hline 8 ou mais & 22,3 & 80,1 & & 60,7 & \\
\hline \multicolumn{6}{|l|}{ Saúde bucal } \\
\hline Necessidade de tratamento autorreferida & & & $<0,001$ & & $<0,001$ \\
\hline \multicolumn{6}{|l|}{ (necessidade de usar ou trocar a dentadura) } \\
\hline Não & 51,1 & 87,0 & & 53,8 & \\
\hline Sim & 46,8 & 68,0 & & 85,2 & \\
\hline Não sei/Não respondeu & 2,1 & 93,9 & & 61,9 & \\
\hline Local de atendimento na última consulta & & & $<0,001$ & & 0,012 \\
\hline \multicolumn{6}{|l|}{ odontológica } \\
\hline Público & 23,9 & 64,0 & & 80,3 & \\
\hline Particular/Convênio & 58,1 & 84,0 & & 63,0 & \\
\hline Nunca foi ao dentista & 16,4 & 80,0 & & 72,0 & \\
\hline Não informado & 1,7 & 66,5 & & 72,0 & \\
\hline
\end{tabular}

No Brasil, por haver grande heterogeneidade socioeconômica entre as macrorregiões, há diferenças na distribuição das perdas dentárias 16 e, consequentemente, no uso e necessidade de prótese dentária conforme mostram os resultados deste estudo. Destaca-se o contraste entre as macrorregiões Sul e Nordeste. A primeira, que é considerada uma das macrorregiões com os melhores índices socioeconômicos do país 21 , apresentou maior prevalência de uso e menor prevalência de necessidade de prótese. Situação oposta à encontrada na Região Nordeste, onde se concentra a menor a prevalência de uso e a maior de necessidade de prótese dentária. Estudos nacionais anteriores já mostravam acentuado contraste entre as macrorregiões do Brasil 22,23, e embora tenha havido redução considerável das desigualdades no acesso e aumento da utilização de serviços odontológicos no Brasil entre 1998 e 2008, algumas inequidades ainda permanecem marcantes entre as macrorregiões 24.

Uma possível explicação para o maior uso de prótese encontrado na Região Sul do país pode estar relacionada ao uso de serviços odontológicos. Levantamentos nacionais anteriores revelaram que a prevalência do uso de serviço odontológico pelo SUS na Região Sul foi inferior a 10\% 22, e que esta foi 


\section{Tabela 3}

Modelos de regressão de Poisson bruto e ajustado para associação entre fatores individuais e prevalência de uso de prótese em idosos de 65-74 anos. SBBrasil $2010(\mathrm{~N}=7.496)$.

\begin{tabular}{|c|c|c|c|c|}
\hline \multirow[t]{2}{*}{ Variável } & \multicolumn{4}{|c|}{ Prevalência de uso de prótese } \\
\hline & RP bruta & IC95\% & RP ajustada & IC95\% \\
\hline \multicolumn{5}{|c|}{ Características socioeconômicas e demográficas } \\
\hline \multicolumn{5}{|l|}{ Sexo } \\
\hline Masculino & 1,00 & & 1,00 & \\
\hline Feminino & 1,23 & $1,12-1,34$ & 1,19 & $1,10-1,29$ \\
\hline \multicolumn{5}{|l|}{ Raça/Cor } \\
\hline Branca & 1,00 & & 1,00 & \\
\hline Preta & 0,77 & $0,67-0,89$ & 0,83 & $0,74-0,93$ \\
\hline Amarela & 0,87 & $0,74-1,03$ & 1,00 & $0,83-1,21$ \\
\hline Parda & 0,90 & $0,84-0,97$ & 0,93 & $0,87-0,99$ \\
\hline Indígena & 0,92 & $0,65-1,30$ & 0,93 & $0,71-1,21$ \\
\hline \multicolumn{5}{|l|}{ Renda familiar (em Reais) } \\
\hline$\leq 500$ & 1,00 & & 1,00 & \\
\hline $501-1.500$ & 1,14 & $0,95-1,37$ & 1,09 & $0,96-1,25$ \\
\hline $1.501-2.500$ & 1,19 & $0,98-1,95$ & 1,10 & $0,96-1,26$ \\
\hline$>2.500$ & 1,15 & $0,93-1,40$ & 1,03 & $0,88-1,20$ \\
\hline \multicolumn{5}{|l|}{ Anos de estudos } \\
\hline Até 4 & 1,00 & & 1,00 & \\
\hline $5-7$ & 1,12 & $1,03-1,23$ & 1,11 & $1,03-1,19$ \\
\hline 8 ou mais & 1,05 & $0,95-1,16$ & 1,03 & $0,94-1,12$ \\
\hline \multicolumn{5}{|l|}{ Saúde bucal } \\
\hline \multicolumn{5}{|c|}{ Necessidade de tratamento autorreferida } \\
\hline Não & 1,00 & & 1,00 & \\
\hline Sim & 0,78 & $0,73-0,84$ & 0,81 & $0,76-0,87$ \\
\hline Não sei/Não respondeu & 1,08 & $1,02-1,14$ & 1,06 & $0,98-1,14$ \\
\hline \multicolumn{5}{|c|}{ Uso/Local de atendimento na última consulta odontológica } \\
\hline Público & 1,00 & & 1,00 & \\
\hline Particular & 1,32 & $1,16-1,52$ & 1,23 & $1,10-1,38$ \\
\hline Plano de saúde/Convênio & 1,25 & $1,06-1,48$ & 1,16 & $1,00-1,36$ \\
\hline Outros & 1,21 & $0,97-1,51$ & 1,25 & $1,05-1,50$ \\
\hline Nunca foi ao dentista & 1,24 & $1,07-1,43$ & 1,19 & $1,04-1,35$ \\
\hline Não informado & 1,04 & $0,68-1,60$ & 1,22 & $1,05-1,42$ \\
\hline
\end{tabular}

IC95\%: intervalo de 95\% de confiança; RP: razão de prevalência.

a região com o menor percentual em relação a nunca ter visitado um dentista 23 . Nossos achados também mostram que é na Região Sul onde se observa maior uso de prótese dentária do tipo ponte fixa, prótese destinada a substituir um ou mais dentes, apoiada em dentes vizinhos ao espaço desdentado. Essas próteses geralmente são confeccionadas nos serviços particulares e apresentam custo elevado em relação aos outros tipos de próteses, sendo, portanto, mais frequentemente usadas em regiões onde há maior nível de desenvolvimento socioeconômico e onde a população tem maior poder de compra por estes serviços.

Desigualdades étnico-raciais têm sido evidenciadas em diversos estudos e são objeto de investigação 25, principalmente no campo da saúde pública. Neste trabalho, pretos e pardos apresentaram uma menor prevalência no uso de prótese quando comparados aos brancos, e não houve diferença com relação à necessidade de prótese, demonstrando desvantagem e exclusão em relação ao uso e acesso a serviços mais complexos e mais caros, como a reabilitação protética 26 . As mulheres apresentaram maior prevalência de uso e menor necessidade de prótese dentária, provavelmente porque costumam 
Tabela 4

Modelos de regressão de Poisson bruto e ajustado para associação entre fatores individuais e prevalência de necessidade de prótese em idosos de 65-74 anos. SBBrasil 2010 ( $N=7.496)$.

\begin{tabular}{|c|c|c|c|c|}
\hline \multirow[t]{2}{*}{ Variável } & \multicolumn{4}{|c|}{ Prevalência de necessidade de prótese } \\
\hline & RP bruta & IC95\% & RP ajustada & IC95\% \\
\hline \multicolumn{5}{|c|}{ Características socioeconômicas e demográficas } \\
\hline \multicolumn{5}{|c|}{ Sexo } \\
\hline Masculino & 1,00 & & 1,00 & \\
\hline Feminino & 0,90 & $0,83-0,97$ & 0,92 & $0,85-1,00$ \\
\hline \multicolumn{5}{|l|}{ Raça/Cor } \\
\hline Branca & 1,00 & & 1,00 & \\
\hline Preta & 1,18 & $1,06-1,31$ & 1,07 & $0,94-1,22$ \\
\hline Amarela & 1,13 & $0,91-1,39$ & 0,99 & $0,80-1,22$ \\
\hline Parda & 1,09 & $0,99-1,20$ & 1,01 & $0,92-1,11$ \\
\hline Indígena & 0,57 & $0,21-1,51$ & 0,51 & $0,22-1,19$ \\
\hline \multicolumn{5}{|l|}{ Renda familiar (em Reais) } \\
\hline$\leq 500$ & 1,00 & & 1,00 & \\
\hline $501-1.500$ & 0,90 & $0,80-1,02$ & 0,93 & $0,83-1,03$ \\
\hline $1.501-2.500$ & 0,81 & $0,66-0,99$ & 0,96 & $0,70-1,07$ \\
\hline$>2.500$ & 0,66 & $0,54-0,81$ & 0,73 & $0,57-0,93$ \\
\hline \multicolumn{5}{|l|}{ Anos de estudos } \\
\hline Até 4 & 1,00 & & 1,00 & \\
\hline $5-7$ & 0,97 & $0,84-1,12$ & 1,00 & $0,89-1,13$ \\
\hline 8 ou mais & 0,85 & $0,74-0,97$ & 0,91 & $0,79-1,04$ \\
\hline \multicolumn{5}{|l|}{ Saúde bucal } \\
\hline \multicolumn{5}{|c|}{ Necessidade de tratamento autorreferida } \\
\hline Não & 1,00 & & 1,00 & \\
\hline Sim & 1,58 & $1,39-1,80$ & 1,58 & $1,39-1,79$ \\
\hline Não sei/Não respondeu & 1,15 & $1,79-1,67$ & 1,14 & $0,79-1,04$ \\
\hline \multicolumn{5}{|c|}{ Uso/Local de atendimento na última consulta odontológica } \\
\hline Público & 1,00 & & 1,00 & \\
\hline Particular & 0,78 & $0,68-0,88$ & 0,87 & $0,77-0,98$ \\
\hline Plano de saúde/Convênio & 0,83 & $0,68-0,99$ & 0,91 & $0,76-1,10$ \\
\hline Outros & 0,90 & $0,72-1,13$ & 0,98 & $0,82-1,19$ \\
\hline Nunca foi ao dentista & 0,90 & $0,77-1,04$ & 0,98 & $0,85-1,12$ \\
\hline Não informado & 0,90 & $0,62-1,28$ & 0,83 & $0,56-1,22$ \\
\hline
\end{tabular}

IC95\%: intervalo de 95\% de confiança; RP: razão de prevalência.

fazer consultas odontológicas com maior regularidade do que os homens e por questões relacionadas ao autocuidado que são mais marcantes no sexo feminino 27.

Conhecer como cada indivíduo percebe a própria saúde é um importante passo para se compreender o padrão de procura por um serviço de saúde, o que deve ser usado para influenciar as tomadas de decisões das políticas públicas 28 . No presente estudo, dentre os que responderam não necessitar usar ou trocar a prótese, 53,8\% necessitavam de acordo com o exame clínico realizado pelo cirurgião-dentista. $\mathrm{Na}$ avaliação do examinador qualquer ausência dentária que afetasse a função ou a estética foi considerada, assim como quando a prótese necessitava ser trocada por falta de retenção, estabilidade, fixação ou prejuízo estético. Se por um lado o emprego do índice apropriado permite a identificação objetiva da necessidade normativa do uso de prótese dentária, por outro a adoção destes índices não leva em conta a efetiva demanda (necessidade subjetiva) do indivíduo pelo uso destas próteses. Essa necessidade subjetiva é influenciada não apenas pela ausência de elementos dentários, mas principal- 
mente por fatores como idade do paciente, conforto, custo do tratamento, preferências individuais, diferenças culturais e acessibilidade aos serviços de saúde ${ }^{29}$. Os casos nos quais as necessidades subjetiva e normativa mais se aproximam são aqueles com grande número de perdas dentárias 29 , o que ocorre na população idosa investigada neste trabalho.

Em todas as macrorregiões, o uso de prótese foi maior no arco superior e as prótese dentária mais utilizadas foram a prótese total e prótese parcial removível que substituem todos os elementos e mais de um elemento, respectivamente. O mesmo padrão se repete quanto à necessidade de prótese dentária. Sabe-se que a progressão da cárie dentária e a perda de dentes ao longo da vida são maiores nos dentes posteriores superiores e menores nos dentes mandibulares anteriores 30 . Um estudo realizado nessa mesma população de idosos mostrou que quanto maior o número de dentes que necessitam ser substituídos maior é o impacto negativo na qualidade de vida relacionada à saúde bucal. Perda de dentes anteriores tem maior impacto em atividades sociais, já a perda dos dentes posteriores afeta mais os domínios funcionais, como comer e falar 9.

Neste estudo, com relação ao uso do serviço odontológico pelos idosos, um maior uso de prótese e uma menor necessidade de prótese ocorreram entre os usuários do serviço particular quando comparados aos usuários do serviço público. Curiosamente, idosos que declararam nunca ter visitado o dentista apresentaram maior uso de prótese do que aqueles usuários do serviço público. Isso pode ter ocorrido por dois motivos. Inicialmente, apesar de ilegal, é comum no Brasil a confecção de próteses dentárias diretamente em laboratórios, por profissionais não habilitados a atender pacientes, como técnicos em prótese dentária ou em laboratórios clandestinos. A segunda hipótese é que, por usarem prótese total por muito tempo, resultado do modelo de atenção mutilador que prevalecia no país no passado, os idosos não acessem serviços odontológicos há bastante tempo, levando os mesmos a responder que nunca foram ao dentista. Assim como a confecção de próteses dentárias, a extração dos dentes desses indivíduos idosos pode ter sido realizada por "práticos" não formados, comuns em certas regiões do país, principalmente no século passado, bem como podem ter ocorrido perdas por doença periodontal avançada sem necessidade de extração.

Até hoje a população idosa é a que apresenta o maior número de necessidades odontológicas acumuladas ${ }^{31}$, revelando o que, historicamente, caracterizou a prestação da atenção odontológica, com ações de baixa complexidade e na sua maioria curativas e mutiladoras 17 . A fluoretação das águas de abastecimento público e a incorporação de fluoretos nos dentifrícios são mecanismos eficazes para a prevenção da ocorrência de cárie dentária 32 , maior responsável pela perda dos dentes. No entanto, essas medidas podem não ter beneficiado os idosos avaliados neste levantamento nacional, pois no momento da implantação dessas políticas grande parte das perdas dentárias já poderiam ter acontecido nesses indivíduos 16. De forma complementar, com base nos resultados do SBBrasil 2003 que revelou prevalência de $75 \%$ de idosos e $30 \%$ de adultos desdentados 12 e com o lançamento da Política Nacional de Saúde Bucal em 2004, o Governo Federal vem aumentando muito os investimentos com intuito de ampliar o acesso da população ao tratamento odontológico, incluindo a reabilitação protética por meio de próteses totais ou parciais removíveis por intermédio dos CEO e dos LRPD ${ }^{17}$. É provável que futuros levantamentos nacionais de saúde bucal possam obter uma melhor dimensão do impacto dessas políticas públicas.

Os resultados deste trabalho ajudam compreender, em parte, o agravo edentulismo em nível nacional, servindo para estimar a gravidade do problema e para subsidiar ações de planejamento. Além disso, a análise dos dados levou em consideração a estrutura complexa da amostragem por conglomerados e os pesos amostrais, sendo estes os principais pontos fortes do estudo. Por outro lado, deve-se levar em consideração que o delineamento transversal não permite o estabelecimento da direção das associações encontradas, impossibilitando a inferência de relações causais. O índice utilizado, uso e necessidade de prótese dentária, também apresenta limitações já que não permite avaliarmos a qualidade das perdas dentárias, como o número de dentes e suas posições nos arcos dentários. A realização de levantamentos epidemiológicos nacionais em um país como o Brasil impõe uma série de desafios logísticos, como a necessidade de contar com um grande número de examinadores com padronização aceitável. Nesse sentido, um número grande de equipes de treinamento foi formado para garantir o processo de calibração dos examinadores e a obtenção de parâmetros de concordância confiáveis.

A redução da ocorrência das doenças bucais mais prevalentes entre os indivíduos mais jovens observada nos levantamentos nacionais, aliada à maior oferta de serviços públicos de saúde bucal e à 
prática mais direcionada à promoção da saúde, permitem vislumbrar que no futuro o uso e a necessidade de prótese nos idosos possam ser menos dramáticos. Essa expectativa deve ser confirmada nos próximos levantamentos nacionais que deverão ter garantida sua ocorrência periódica para o monitoramento da saúde bucal no país. Considerando-se o grave quadro observado em relação à necessidade de prótese e o seu uso na população idosa estudada, ainda é importante reafirmar a necessidade da ampliação da oferta de serviços especializados para a reabilitação protética dentro do SUS.

\section{Colaboradores}

J. S. Azevedo e F. F. Demarco contribuíram no desenho do estudo, análise dos dados e redação do artigo científico. M. S. Azevedo, L. J. C. Oliveira e M. B. Correa colaboraram na análise dos dados e redação do artigo científico.

\section{Agradecimentos}

Os autores agradecem ao Ministério da Saúde, que proporcionou o auxílio financeiro para a realização da Pesquisa Nacional de Saúde Bucal (SBBrasil 2010).

\section{Referências}

1. Beard JR, Officer AM, Cassels AK. The World Report on Ageing and Health. Gerontologist 2016; 56 Suppl 2:S163-6.

2. World Bank. Becoming old in an older Brazil. Washington DC: World Bank; 2011.

3. Colussi CF, Freitas SF. Aspectos epidemiológicos da saúde bucal do idoso no Brasil. Cad Saúde Pública 2002; 18:1313-20.

4. Yellowitz JA, Schneiderman MT. Elder's oral health crisis. J Evid Based Dent Pract 2014; 14 Suppl:191-200.

5. Marcenes W, Kassebaum NJ, Bernabé E, Flaxman A, Naghavi M, Lopez A, et al. Global burden of oral conditions in 1990-2010: a systematic analysis. J Dent Res 2013; 92:592-7.

6. Barbato PR, Nagano HCM, Zanchet FN, Boing AF, Peres MA. Perdas dentárias e fatores sociais, demográficos e de serviços associados em adultos brasileiros: uma análise dos dados do Estudo Epidemiológico Nacional (Projeto SB Brasil 2002-2003). Cad Saúde Pública 2007; 23:1803-14.

7. Seerig LM, Nascimento GG, Peres MA, Horta BL, Demarco FF. Tooth loss in adults and income: systematic review and meta-analysis. J Dent 2015; 43:1051-9.

8. De Marchi RJ, Leal AF, Padilha DM, Brondani MA. Vulnerability and the psychosocial aspects of tooth loss in old age: a Southern Brazilian study. J Cross Cult Gerontol 2012; 27:239-58.

9. Azevedo MS, Correa MB, Azevedo JS, Demarco FF. Dental prosthesis use and/or need impacting the oral health-related quality of life in Brazilian adults and elders: results from a national survey. J Dent 2015; 43:1436-41. 
10. Peres MA, Tsakos G, Barbato PR, Silva DA, Peres KG. Tooth loss is associated with increased blood pressure in adults: a multidisciplinary population-based study. J Clin Periodontol 2012; 39:824-33.

11. Abnet CC, Qiao YL, Dawsey SM, Dong ZW Taylor PR, Mark SD. Tooth loss is associated with increased risk of total death and death from upper gastrointestinal cancer, heart disease, and stroke in a Chinese population-based cohort. Int J Epidemiol 2005; 34:467-74.

12. Martins AMEBL, Barreto SM, Pordeus IA. Características associadas ao uso de serviços odontológicos entre idosos dentados e edentados no Sudeste do Brasil: Projeto SB Brasil. Cad Saúde Pública 2008; 24:81-92.

13. Peres MA, Bastos JL, Watt RG, Xavier AJ, Barbato PR, D'Orsi E. Tooth loss is associated with severe cognitive impairment among older people: findings from a population-based study in Brazil. Aging Ment Health 2015; 19:876-84.

14. Montero J, Castillo-Oyagüe R, Lynch CD, Albaladejo A, Castaño A. Self-perceived changes in oral health-related quality of life after receiving different types of conventional prosthetic treatments: a cohort follow-up study. J Dent 2013; 41:493-503.

15. Fédération Dentaire Internationale. Global goals for oral health in the year 2000. Int Dent J 1982; 32:74-7.

16. Peres MA, Barbato PR, Reis SC, Freitas CH, Antunes JL. Perdas dentárias no Brasil: análise da Pesquisa Nacional de Saúde Bucal 2010. Rev Saúde Pública 2013; 47 Suppl 3:S78-89.

17. Pucca GA, Gabriel M, de Araujo ME, de Almeida FC. Ten years of a National Oral Health Policy in Brazil: innovation, boldness, and numerous challenges. J Dent Res 2015; 94: 1333-7.

18. Roncalli AG, Silva NN, Nascimento AC, Freitas CHSM, Casotti E, Peres KG, et al. Aspectos metodológicos do Projeto SBBrasil 2010 de interesse para inquéritos nacionais de saúde. Cad Saúde Pública 2012; 28 Suppl:S40-57.

19. World Health Organization. Oral Health Survey: basic methods. Geneva: World Health Organization; 1987.

20. Thomson WM. Social inequality in oral health. Community Dent Oral Epidemiol 2012; 40 Suppl 2:28-32.

21. Peiter PC. Condiciones de vida, situación de la salud y disponibilidad de servicios de salud en la frontera de Brasil: un enfoque geográfico. Cad Saúde Pública 2007; 23 Suppl 2:S237-50.

22. Peres MA, Iser BP, Boing AF, Yokota RT, Malta DC, Peres KG. Desigualdades no acesso e na utilização de serviços odontológicos no Brasil: análise do Sistema de Vigilância de Fatores de Risco e Proteção para Doenças Crônicas por Inquérito Telefônico (VIGITEL 2009). Cad Saúde Pública 2012; 28 Suppl:S90-100.
23. Matos DL, Giatti L, Lima-Costa MF. Fatores sócio-demográficos associados ao uso de serviços odontológicos entre idosos brasileiros: um estudo baseado na Pesquisa Nacional por Amostra de Domicílios. Cad Saúde Pública 2004; 20:1290-7.

24. Peres KG, Peres MA, Boing AF, Bertoldi AD, Bastos JL, Barros AJ. Reduction of social inequalities in utilization of dental care in Brazil from 1998 to 2008. Rev Saúde Pública 2012; 46:250-8.

25. Bastos JL, Celeste RK, Faerstein E, Barros AJ. Racial discrimination and health: a systematic review of scales with a focus on their psychometric properties. Soc Sci Med 2010; 70: 1091-9.

26. Bastos JL, Peres MA, Peres KG, Dumith SC, Gigante DP. Diferenças socioeconômicas entre autoclassificação e heteroclassificação de cor/ raça. Rev Saúde Pública 2008; 42:324-34.

27. Machado LP, Camargo MB, Jeronymo JC, Bastos GA. Regular use of dental services among adults and older adults in a vulnerable region in Southern Brazil. Rev Saúde Pública 2012; 46:526-33.

28. Moreira RS, Nico LS, Sousa MLR. Fatores associados à necessidade subjetiva de tratamento odontológico em idosos brasileiros. Cad Saúde Pública 2009; 25:2661-71.

29. Narby B, Kronstrom M, Soderfeldt B, Palmqvist S. Prosthodontics and the patient: what is oral rehabilitation need? Conceptual analysis of need and demand for prosthodontic treatment. Part 1: a conceptual analysis. Int J Prosthodont 2005; 18:75-9.

30. Broadbent JM, Thomson WM, Poulton R. Progression of dental caries and tooth loss between the third and fourth decades of life: a birth cohort study. Caries Res 2006; 40: 459-65.

31. Fonesca FA, Jones KM, Mendes DC, dos Santos Neto PE, Ferreira RC, Pordeus IA, et al. The oral health of seniors in Brazil: addressing the consequences of a historic lack of public health dentistry in an unequal society. Gerodontology 2015; 32:18-27.

32. Narvai PC, Frazão P, Roncalli AG, Antunes JL. Cárie dentária no Brasil: declínio, polarização, iniqüidade e exclusão social. Rev Panam Salud Pública 2006; 19:385-93. 


\section{Abstract}

The objectives of this study were to evaluate the use of and need for dental prostheses among elderly Brazilians (65-74 years of age) and to verify associated factors. Data were analyzed from 7,496 elderly participants in the National Oral Health Survey in 2010 (SBBrazil 2010). Use of and need for dental prosthesis were the outcomes. The exposure variables included demographic and socioeconomic characteristics, dental services use, and self-rated oral health. Descriptive, bivariate, and multivariate analyses were performed. Prevalence rates for use of and need for dental prostheses were $78.2 \%$ and $68.7 \%$, respectively. The Southeast Region had the highest prevalence of use (71.3\%) and the greatest need for dental prostheses (82.9\%). Multivariate Poisson regression analyses showed greater use of dental prostheses by women, individuals with 5 to 7 years of schooling, and users of private dental services, and lower use by black individuals and those with self-reported need for dental prostheses. Less need for dental prostheses was seen in women and in users of private services, and greater need in those with self-reported need for dental prostheses. Regional differences were seen in the distribution of use and need for dental prostheses. Still, the findings showed high prevalence rates for both outcomes in all regions of Brazil. Socioeconomic and demographic variables and use of dental services influenced the use of and need for dental prostheses.

Dental Prosthesis; Health Surveys; Oral Health; Aged

\section{Resumen}

El objetivo de este estudio fue evaluar el uso y la necesidad de prótesis dentales entre los ancianos brasileiros (65-74 años) y verificar sus factores asociados. Se analizaron datos de 7.496 ancianos participantes en la Encuesta Nacional de Salud Bucal, realizada en 2010 (SBBrasil 2010). El uso y la necesidad de prétesis dentales se usaron como resultados. Las variables de exposición incluyeron características demográficas, socioeconómicas, de uso de servicios y de autopercepción de salud bucal. Se realizaron análisis descriptivos, bivariados y multivariados. La prevalencia de uso y necesidad de prótesis dentales fue de un 78,2\% y 68,7\%, respectivamente. La región nordeste fue la que presentó la menor prevalencia de uso $(71,3 \%)$ y la mayor de necesidad prótesis dentales (82,9\%). Los análisis multivariados, mediante la regresión de Poisson, revelaron un mayor uso de prótesis dentales en mujeres, en aquellos con 5 a 7 años de estudios y en los que fueron a servicios privados, $y$ hubo un menor uso en individuos negros y con necesidad autorreferida de prótesis dentales. Se observó una menor necesidad de prótesis dentales en mujeres y en los usuarios de servicios particulares, y mayor en aquellos que autoinformaron una necesidad de prótesis dentales. Se observaron diferencias regionales en la distribución del uso y necesidad de prótesis dentales. Incluso así, los hallazgos revelaron altas prevalencias de ambos en los resultados en todas las regiones. Variables socioeconómicas, demográficas y de uso de servicios influenciaron la ocurrencia de uso y necesidad de prótesis dentales.

Prótesis Dental; Encuestas de Salud; Salud Bucal; Anciano
Recebido em 07/Abr/2016

Versão final reapresentada em 29/Jul/2016 Aprovado em 22/Set/2016 